Supporting information for

\title{
Highly Transparent Covalent Mucin Coatings Improve the Wettability and Tribology of Hydrophobic Contact Lenses
}

Carolin A. Rickert ${ }^{1}$, Barbara Wittmann ${ }^{1}$, Roland Fromme ${ }^{2}$ and Oliver Lieleg ${ }^{1, *}$

${ }^{1}$ Department of Mechanical Engineering and Munich School of Bioengineering, Technical University of Munich, 85748 Garching b. München, Germany

${ }^{2}$ Woehlk Contactlinsen GmbH, 24232 Schönkirchen, Germany

* Corresponding Author: oliver.lieleg@tum.de. 
S1 - Characterization of the tribological setup. For the tribological experiments, a customized setup was used consisting of a silicone-based contact lens holder (carrying the respective lens) and a full porcine eye that was fixed in a custom-made holder (see Methods). Based on the applied normal force of $F_{\mathrm{N}}=0.14 \mathrm{~N}$, the resulting contact pressure $p_{0}$ between the contact lens and the eye surface can be estimated according to the Hertzian model ${ }^{1}$ as described below in equation S1:

$$
p_{0}=\frac{2}{3} p_{\max }=\frac{2}{3 \pi} \sqrt[3]{6 F_{N} *\left(E^{\prime} \rho\right)^{2}} \quad \text { with } \quad \frac{1}{E^{\prime}}=\frac{1-v_{1}^{2}}{E_{1}}+\frac{1-v_{2}^{2}}{E_{2}} \quad \text { and } \quad \rho=\frac{1}{r_{1}}+\frac{1}{r_{2}}
$$

Here, the radii of the contact lens holder $\left(r_{1}=7.35 \mathrm{~mm}\right)$ and the eye $\left(r_{2}=10.25 \mathrm{~mm}\right)$ were used to describe the setup curvatures. Furthermore, we approximated the Young's modulus $E_{1}$ and the Poisson's ratio $v_{1}$ of the contact lens holder with the respective values of pure PDMS ( $E_{\mathrm{PDMS}}=2$ $\left.\mathrm{MPa}, \nu_{\mathrm{PDMS}}=0.49\right)^{2}$. Then, together with the Young's modulus and Poisson's ratio of porcine eyes $\left(E_{2}=0.2 \mathrm{MPa}, v_{2}=0.5\right)^{3}$, the Hertzian contact theory returned an average contact pressure of approximately $30 \mathrm{kPa}$ and a radius of the contact area of $r_{\mathrm{c}}=1.23 \mathrm{~mm}-$ the latter of which agrees well with our visual impression from the tribological measurements. With an average sliding velocity $v_{\mathrm{s}}=0.125 \mathrm{~mm} \mathrm{~s}^{-1}$ (estimated from the rotational speed used in the measurements at a radius of $0.615 \mathrm{~mm}$ ), a lubricant viscosity of $\eta_{b u f f e r}=1 \mathrm{mPas}$ and the contact pressure determined above, we can also estimate a Sommerfeld number of $S=\frac{v_{S} * \eta_{\text {buffer }}}{p_{0}}=4.11 \times 10^{-12}$ for our system.

Moreover, as we used a custom-made setup on a commercial rheometer, the measured friction coefficients were by default calculated under the assumption of a ball-on-three-pins geometry. To transfer those values to the ocular setup, we applied a correction factor to the determined friction coefficients as follows (for further information see user manual of the rheometer):

$$
\mu_{\text {corrected }}=\mu_{\text {measured }} * \frac{3 * r_{\text {ball }} * \sin (\alpha)}{2 * r_{c} * \cos (\alpha)}
$$


S2 - Mathematical definition of the metrological surface parameters. In the main paper, we quantified the wear formation on the corneal surfaces by calculating relative surface parameters that compare the surface before and after tribological treatment. In the following, the metrological parameters used in the main paper are explained in more detail. In all equations shown below, $A$ denotes the definition area with the horizontal coordinates being $x$ and $y . Z$ represents the ordinal height values of each point measured from the mean height.

First, we determined the root mean square height, $S_{\mathrm{q}}$ - which is equivalent to the standard deviation of the ordinal height - according to equation (S3):

$$
S_{q}=\sqrt{\frac{1}{A} \iint_{A} Z^{2}(x, y) d x d y}
$$

Second, we calculated the developed interfacial area ratio, $S_{\mathrm{dr}}$, as described in equation (S4). This $S_{\mathrm{dr}}$ value denotes the percentage of surface increase resulting from the texture in comparison to a completely planar surface:

$$
S_{d r}=\frac{1}{A}\left[\iint_{A}\left(\sqrt{\left[1+\left(\frac{\partial z(x, y)}{\partial x}\right)^{2}+\left(\frac{\partial z(x, y)}{\partial y}\right)^{2}\right]}-1\right) d x d y\right]
$$

Third, we analyzed the ten-point-height, $S_{10 z}$, which takes into account the height of the five highest local maxima above the mean surface and the depth of the five lowest local minima below the mean height (eq. S5):

$$
S_{10 z}=\frac{1}{10}\left(\sum_{i=1}^{5} Z_{i, \max }+\sum_{j=1}^{5} Z_{j, \min }\right)
$$

Last, we assessed the peak material volume, $V_{\mathrm{mp}}$, which represents the material volume at an areal material ratio of $p=10 \%$ as depicted in Figure S1: 


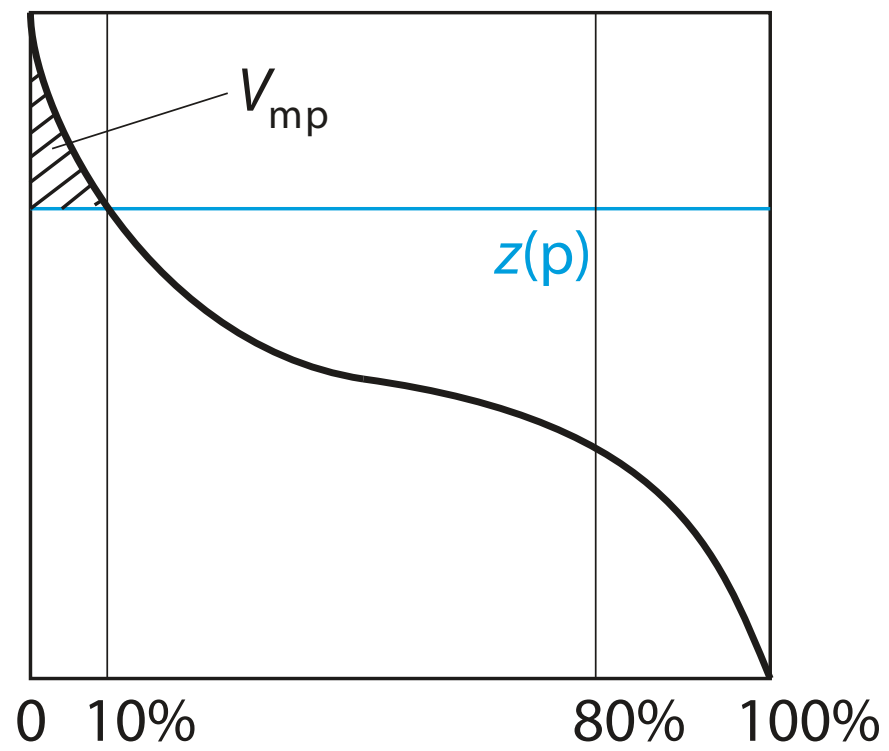

Figure S1. Diagram depicting the volumetric ISO 25178 parameter $V \mathrm{mp}$. The vertical line $z(p)$ represents the chosen threshold value from which the volumetric parameter $V_{\mathrm{mp}} \quad$ was determined (see the main text for details).

S3 - Negative control for the ELISA. A negative control was performed to ensure that the fluorescence signals obtained during the ELISA measurements do not originate from primary antibodies that are trapped within the lens material but only from mucin molecules that adhere to the lens surface. Therefore, the ELISA was performed with contact lenses that were either left uncoated or covalently coated with MUC5AC. The resulting fluorescence intensities were determined and normalized regarding the intensities obtained for a completely empty well (see Figure S2). When no primary antibody was added, we did not observe increased fluorescence intensities - neither for the uncoated sample, nor for the sample coated with MUC5AC. By adding the primary antibody, however, a strong increase of the fluorescence intensity is observable. Hence, the ELISA as performed in our study specifically and reliably detects mucin molecules that are present on the lens surface. 


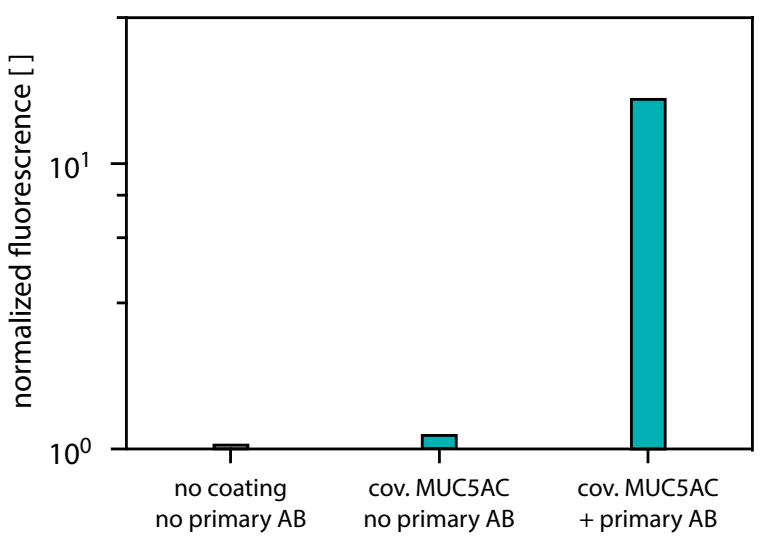

Figure S2. Normalized fluorescence intensities obtained during ELISA measurements. The intensities were obtained with and without adding the primary antibody $(\mathrm{AB})$ during the ELISA procedure with PDMS contact lenses that were either left uncoated or covalently coated with MUC5AC.

S4 - Mucin adhesion onto PDMS-coated QCM-D chips. Mucin adsorption onto hydrophobic PDMS surfaces was quantified using a quartz crystal microbalance with dissipation monitoring (3T analytik, Tuttlingen, Germany). In brief, gold coated quartz sensors (3T-analytik) were spincoated with a thin layer of PDMS as follows: PDMS pre-polymer and cross-linker (Sylgard 184, DowCorning, MI, USA) were mixed in a ratio of 10:1. Then, $10 \mu \mathrm{L}$ of this PDMS premix were added to $1 \mathrm{~mL}$ of $\mathrm{n}$-hexane, and $100 \mu \mathrm{L}$ of this PDMS/n-hexane solution were pipetted onto the center of the gold-coated chip surface while rotating the chip on a spin-coater (WS-650MZ23NPPB, Laurell, North Wales, PA, USA). These rotations were conducted first at $1500 \mathrm{rpm}$ for $20 \mathrm{~s}$ and then at $3000 \mathrm{rpm}$ for $60 \mathrm{~s}$. After drying the samples in an oven at $40{ }^{\circ} \mathrm{C}$ overnight, adsorption measurements were performed at $30{ }^{\circ} \mathrm{C}$. First, the quartz crystals were rinsed with 20

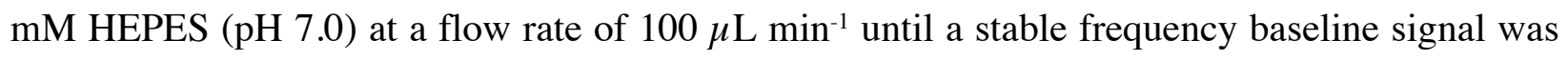
achieved, and this baseline signal was recorded for $15 \mathrm{~min}$. For the adsorption of mucin molecules, 
a $0.01 \%(\mathrm{w} / \mathrm{v})$ mucin solution (solubilized in $20 \mathrm{mM}$ HEPES, $\mathrm{pH} 7.0$ ) was injected into the test chamber and flushed over the PDMS coated sensor at a flow rate of $100 \mu \mathrm{L} \mathrm{min}^{-1}$. The resulting frequency shift, which serves as a measure for the adsorption efficiency of the mucins, was monitored for 45 min (Figure S2) using two commercial softwares (qCell T Q2 1.9 and qGraph Viewer 1.8; both 3t analytik).

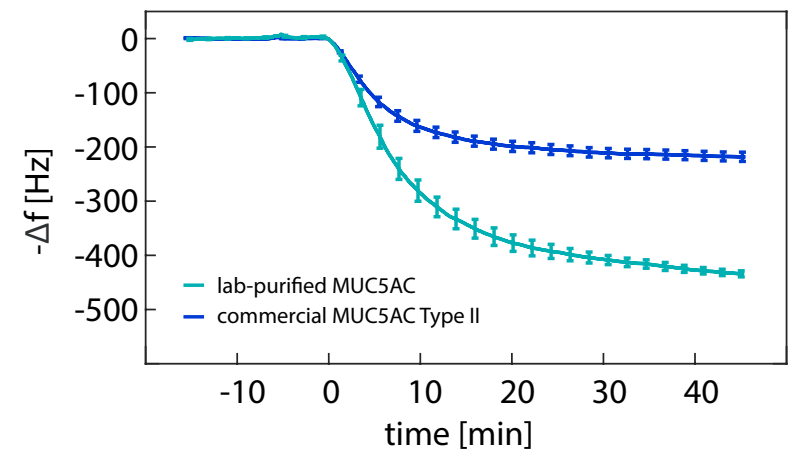

Figure S3. Adsorption of two mucin variants onto hydrophobic PDMS surfaces. The adsorption of manually purified MUC5AC and commercial porcine gastric mucin (Type II, Sigma Aldrich) was quantified by recording frequency shifts over time. The error bars denote the standard error of the mean as obtained from $n \geq 3$ individual measurements. 


\section{S5 - Profilometric images of cornea samples}
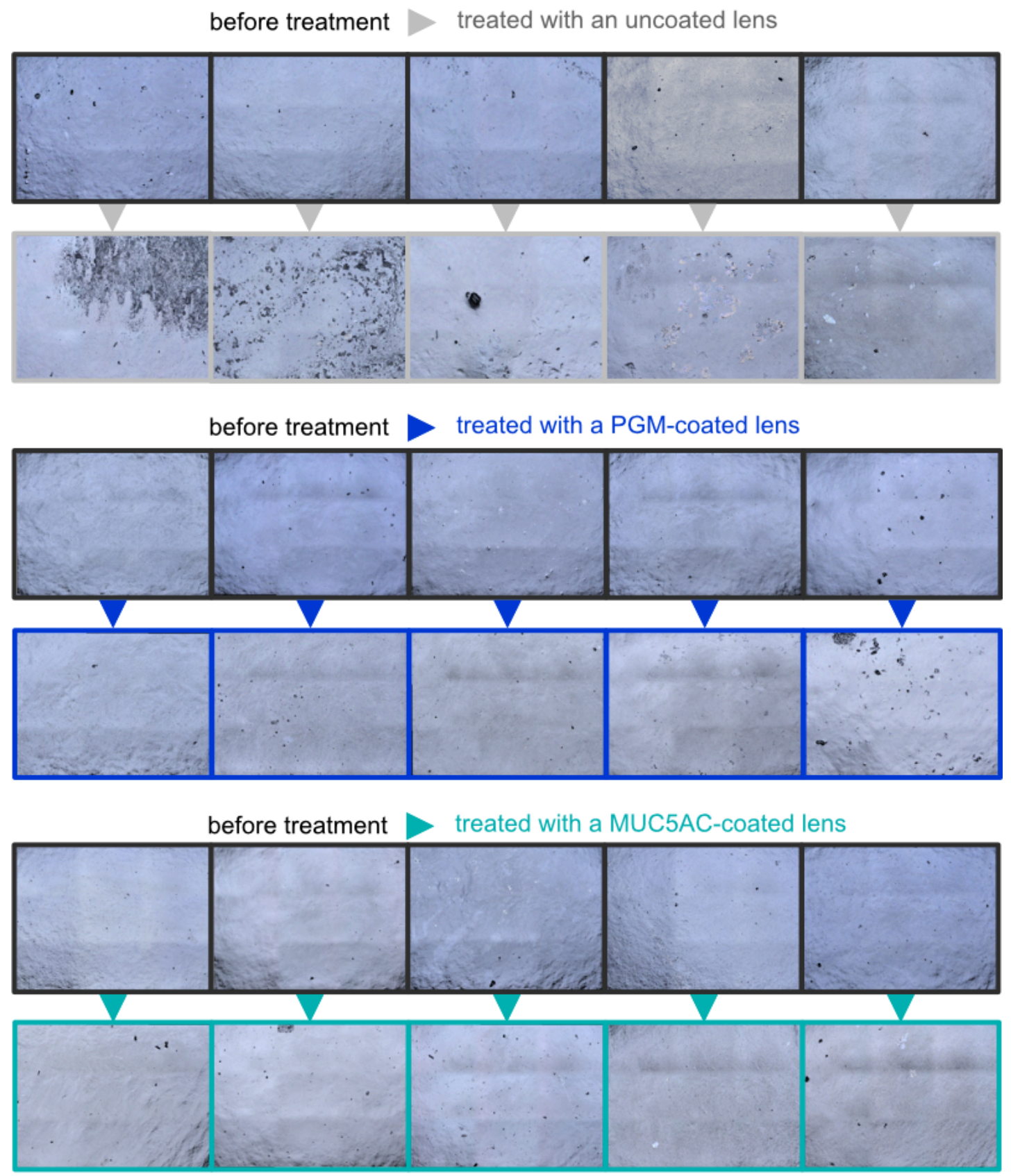

Figure S4. Profilometry images of corneal tissue before and after tribological treatment. The images were obtained before (black boxes) and after tribological treatments using either an uncoated PDMS lens (grey boxes) or PDMS lenses that were covalently coated with commercial PGM (blue) or manually purified MUC5AC (cyan), respectively. 
S6 - Quantification of wear formation by additional ISO parameters.
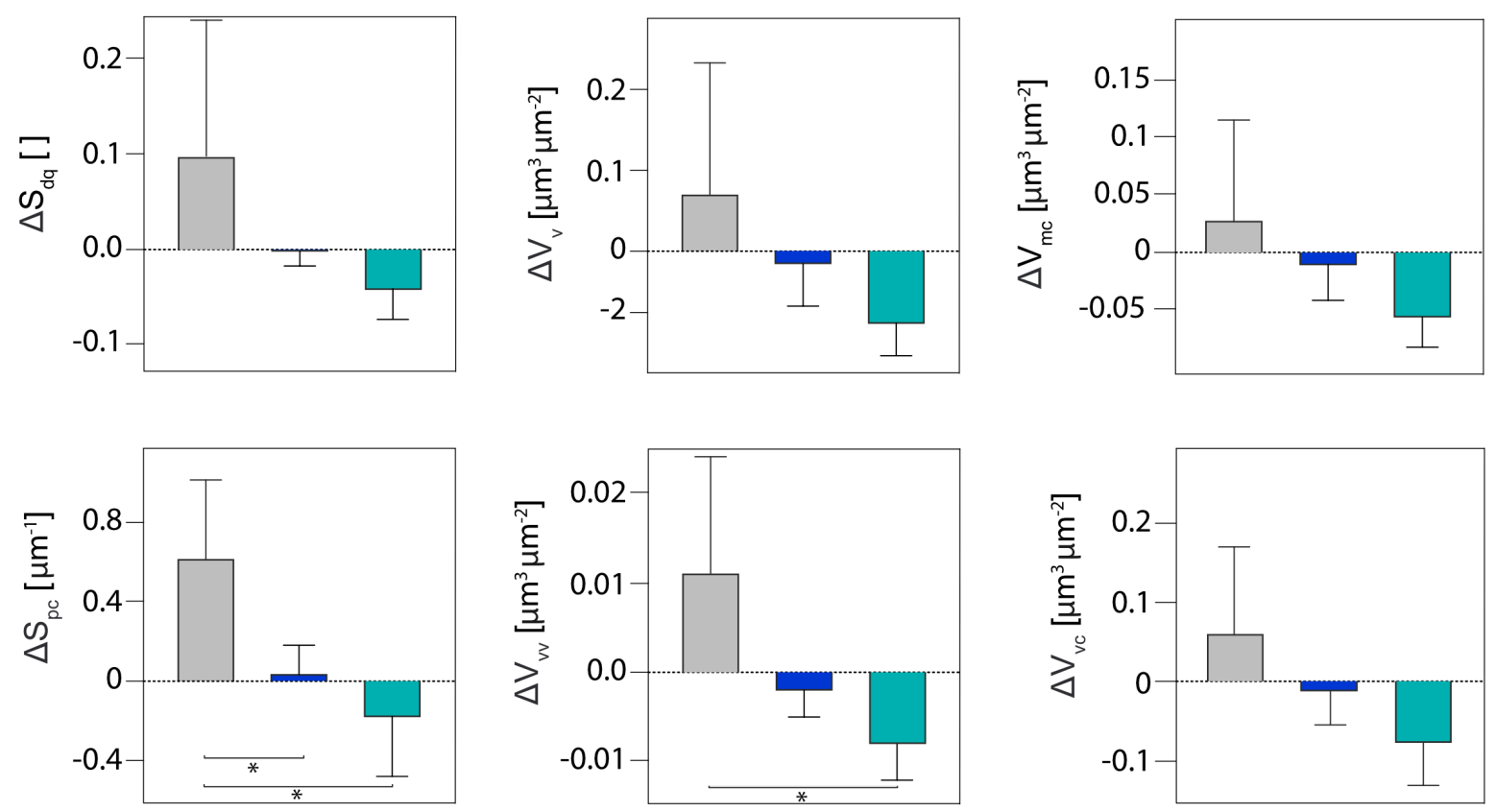

Figure S5. Relative surface roughness parameters of corneal tissue. Six relative surface parameters were determined according to ISO 25178-2 to support the parameters already shown in the main paper. The error bars denote the standard deviation as obtained from $n=5$ independent samples. The asterisks indicate significance at a level of $p=0.05$. 


\section{REFERENCES}

(1) Hertz, H. Über die Berührung fester elastischer Körper. J. reine angew. Math. 1882, 92, $156-171$.

(2) Johnston, I.; McCluskey, D.; Tan, C.; Tracey, M. Mechanical characterization of bulk Sylgard 184 for microfluidics and microengineering. J. Micromech. Microeng. 2014, 24, 035017.

(3) Pierscionek, B. K.; Asejczyk-Widlicka, M.; Schachar, R. A. The effect of changing intraocular pressure on the corneal and scleral curvatures in the fresh porcine eye. $B r . J$. Ophthalmol. 2007, 91, 801-803. 